\section{Identificação e procedência de periódicos nacionais referentes à temática de saúde pública ou saúde coletiva, editados entre 1998 e 2003}

\author{
Identification and origin of Brazilian journals \\ related to public or collective health issues, \\ published from 1998 to 2003
}

Guido Rummler 1

Aracy Witt de Pinho Spinola 2

\author{
1 Departamento de Saúde, \\ Universidade Estadual \\ de Feira de Santana, \\ Feira de Santana, Brasil. \\ 2 Departamento de Prática \\ de Saúde Pública, \\ Faculdade de Saúde Pública, \\ Universidade de São Paulo, \\ São Paulo, Brasil. \\ Correspondência \\ G. Rummler \\ Departamento de Saúde \\ Universidade Estadual \\ de Feira de Santana. \\ $A v$. Sete de setembro \\ 2901/1201, Salvador, BA \\ 40130-000, Brasil. \\ guidorummler@uefs.br
}

\begin{abstract}
The diversity of contents regarding Brazilian scientific journals related to public or collective health reflects peculiar aspects of this field's reach and its interfaces with several other fields. A bibliometric survey was conducted with five databases, including catalogues and sites, aimed at identifying journals related to the public/collective health field and published from 1998 to 2003, while identifying the geographic location of the respective institutions. A total of 118 titles were initially identified, subsequently reduced to 83 journals when indicators of these bases were confronted and matched, 66 of which were published in the last five years. Some 57.6\% of the institutions responsible for these journals are located in the Southeast of Brazil; 16.7\% in the South; $13.6 \%$ in the Central West; $10.6 \%$ in the Northeast; and $1.5 \%$ in the North. The results are displayed in a summary chart according to journal title, ISSN, name of the institution in charge, and respective regional location.
\end{abstract}

Public Health; Periodical Index; Periodicals; Bibliometrics

\section{Introdução}

Mesmo que as denominações Saúde Pública e Saúde Coletiva sejam muitas vezes empregadas de forma indiferenciada, tem-se para as mesmas um leque de abrangência com assuntos e temas próprios da interface destes com muitas outras áreas. Assim é possível localizar estudos referentes à Saúde Pública ou Saúde Coletiva não necessariamente veiculados em publicações cujos títulos se reportem a uma ou outra dessas denominações.

Diante disso, objetivou-se, com o presente trabalho, apresentar um elenco de revistas científicas publicadas nacionalmente, citadas em registros institucionais da Saúde Pública e/ou Coletiva, bem como identificar a entidade responsável por cada uma delas e ilustrar graficamente sua distribuição quanto à área geográfica da instituição de procedência.

Vale considerar que um dos requisitos de identidade para periódicos é o seu registro numérico ou ISSN - International Standard Serial Number-, relacionado com normas técnicas e de padronização. Outra fonte de possível recuperação de informações sobre uma revista é o Catálogo Coletivo Nacional de Publicações Seriadas - CCN - (http://www.ibict.br), que reúne assentamentos de catálogos produzidos pelas principais bibliotecas do país. A identificação de vários títulos de revistas pode ser feita também por intermédio da lista compilada pe- 
la biblioteca eletrônica SciELO - Scienttific Electronic Library Online - (http:/ / www.scielo. org); através do portal Periódicos/CAPES (http: / / www.periodicos.capes.gov.br) da Fundação Coordenação de Aperfeiçoamento de Pessoal de Nível Superior, mas cujo acesso é restrito a instituições de ensino superior; por meio do Índice de Revistas Latino-Americanas em Ciências - Periódica (http://www.dgbiblio.unam.mx/ periodica.html); através do Catálogo de Revistas da Biblioteca Virtual de Saúde - BVS - (http: //revistas.bvs.br) e, também, por meio de outros sites, a exemplo dos selecionados pelo sistema Prossiga (http://www.prossiga.br/portal dereferencia/).

De outra forma, ainda, pode-se identificar revistas por consulta às chamadas Bases de Indexação, onde constam títulos e outros dados referentes às mesmas. A inclusão nelas de um periódico ocorre após aprovação do mesmo frente a critérios de seleção, a exemplo dos citados na base Lilacs - Literatura Latino-Americana e do Caribe em Ciências de Saúde - (http: //www.bireme.br/abd/P/crit_Selecao.htm, 2002), tais como regularidade, periodicidade, normalização, tempo de existência etc. Considerando a área aqui estudada, tem-se para a maioria dos periódicos nacionais, quando indexados, registros na base Lilacs (http://www. bireme.br/abd/P/lista_pais.htm) e/ou Latindex - Sistema Regional de Información em Línea para Revistas Científicas de América Latina, el Caribe, España y Portugal (http://www. latindex.unam.mx/). É apropriado também lembrar da importância que tem nos meios acadêmicos a qualidade atribuída a essas revistas conforme considerada na avaliação da produção científica, que é feita por entidades de fomento à pesquisa ou de avaliação de cursos. Dada a relação da CAPES com esse fato, vale acrescentar às fontes de dados mencionadas a própria lista de classificação de periódicos Qualis-CAPES (http://www2.sede.embrapa.br: 8080/img/pat/capes.htm, acessado em 14/Mar/ 2003).

Por outro lado, se as revistas nacionais são meio importante da divulgação do conhecimento produzido nas áreas em questão, contrapõe-se a esse fato toda uma gama de problemas que envolve a sua produção e, particularmente, o que se relaciona com a regularidade de sua circulação.

Diante disso, desenvolveu-se o presente estudo com o intuito de reunir informações iniciais que contribuam para formulações e análises de aspectos relacionados com o contexto das publicações nacionais referentes à Saúde Pública/Coletiva.

\section{Metodologia}

Para a composição de um quadro informativo contendo os títulos de revistas científicas nacionais referentes à temática Saúde Pública ou Saúde Coletiva, com os respectivos ISSN, e também com os nomes das entidades responsáveis e sua localização regional, foi realizada uma pesquisa utilizando basicamente a recuperação de dados via Internet, complementada por consultas a listagens institucionais, ou por busca física às próprias revistas. Trata-se, portanto, de um estudo exploratório-descritivo, em que foram acessados catálogos e portais de revistas nos quais se encontra maior número de periódicos nacionais.

Para se obter essas informações foram consultadas as seguintes fontes:

- Via portal BIREME - a Lista de Revistas - Títulos Nacionais, do Catálogo de Revistas de Saúde Pública da BVS, para levantamento dos dados: título, ISSN, menção de responsabilidade e respectiva localidade. Com subseqüente acesso ao link Catálogo Sécs, foi recuperado o número/ano mais recente da revista, conforme registro referente às coleções de bibliotecas que alimentam essa base;

- Via portal Latindex - a base Catálogo, sendo efetuadas as buscas de modo análogo ao descrito no item anterior;

- Via portal IBICT - o CCN, para obtenção dos títulos, ISSN, imprenta e registro do volume/ano da edição mais recente, dentre os indicados como existentes em coleções de bibliotecas que informam essa base. Inicialmente, essa busca foi feita com o preenchimento dos campos "assunto: Saúde Pública ou Saúde Coletiva”, "país de publicação: Brasil” e "situação: C - vigente”. Para obtenção de dados de revistas sem referência à Saúde Pública nesse catálogo, mas com essa indicação em alguma das outras fontes consultadas, fez-se a busca por preenchimento do campo referente ao título;

- Via portal BIREME - a Lista de Periódicos/ Brasil indexados na Base de Dados Lilacs, para recuperar títulos de revistas;

- Base de Dados de ISSN - fornecida por correio eletrônico pelo Centro Brasileiro do ISSN, onde foram coletados o título, o ISSN e a imprenta dos periódicos nacionais correntes, nela referentes ao assunto Saúde Pública/Coletiva. Essa base foi também utilizada quanto a revistas concernentes à Medicina, ou Odontologia, ou Nutrição, ou Enfermagem, ou Farmácia, ou Educação Física, ou Fisioterapia, ou Pedagogia, ou Educação, mas que em outras fontes encontravam-se relacionadas à Saúde Pública/Coletiva; 
- Catálogo Geral Qualis-2001, disponibilizado pela CAPES, via correio eletrônico, para verificação da existência, em lista de classificação Qualis-CAPES, dos títulos e respectivos ISSN das revistas identificadas nas fontes anteriores.

Com base nos títulos assim reunidos, foi formulada uma lista única, onde se evitou a duplicidade de entrada de periódicos com título diferenciado, em razão da mudança de denominação em determinada época de sua veiculação, ou de títulos diversos para uma mesma revista, por erro de registro em alguma das bases, ou de variação de ordem e/ou supressão de vocábulos na apresentação dos títulos, ou, ainda, de possíveis equívocos quanto aos demais identificadores apresentados por alguma das fontes pesquisadas. Para isso, fez-se confrontações, cruzamentos e complementações de informações de cada fonte em relação às outras, considerando título, ISSN e entidade responsável. Para o recorte referente ao período de circulação da revista entre 1998 e 2003, foi inicialmente utilizado o CCN, considerando a informação da existência de exemplares da revista no acervo de alguma das bibliotecas que alimentam essa base de dados. Para aquelas não constantes desse catálogo, pesquisou-se, via Sistema Google, a indicação de páginas de Internet relacionadas com o periódico, ou buscou-se o acesso à própria revista para a obtenção dessa informação. O mesmo procedimento foi utilizado para levantamento de algum outro dado que não tivesse registro nas bases mencionadas.

Na Tabela 1 pode-se reconhecer em qual das fontes de dados foi encontrada a revista referente à temática de Saúde Coletiva ou Saúde Pública, e quais fontes a remetem para outra denominação de assunto/especialidade/tema.

Para indicar a distribuição relativa das revistas quanto à procedência regional, estabeleceu-se o porcentual referente à localização, por unidade e região federativa, das respectivas entidades responsáveis. Como suporte à validação metodológica do presente trabalho, foram considerados aspectos e etapas de estratégia de busca referidos por Lopes 1, particularmente quanto ao estabelecimento dos parâmetros relevantes para a pesquisa, identificação das bases de dados quanto aos campos de busca disponibilizados, expansão da busca a campos relacionados e sua operacionalização.

\section{Resultados e discussão}

O processo de busca utilizado para identificar revistas científicas nacionais referentes à Saúde Pública e/ou Saúde Coletiva, e com infor- mação de "vigente" em pelo menos uma das bases de dados consultadas, propiciou inicialmente a recuperação de 118 títulos de periódicos. Após processo de confrontação, cruzamento e complementação dos dados, esse número foi reduzido para 83. Eliminando-se desse elenco aqueles para os quais não foi encontrado indicativo da existência de alguma revista editada dentro do período de 1998 a 2003, foi obtida uma listagem final de 66 títulos.

A identificação das revistas pelo título, ISSN, denominação da entidade responsável e Unidade da Federação em que esta se situa encontra-se na Tabela 1. Fazem parte dessa tabela informações sobre em qual das fontes consultadas a revista se refere à Saúde Pública e/ou Saúde Coletiva, assim como em qual das bases se refere a outra área da saúde. Embora nele se encontre uma diversidade de títulos compreendendo áreas de interface ou campos afins com a Saúde Pública/Coletiva, deve-se considerar que esse quadro foi composto tendo como um dos critérios o fato de que cada revista encontra referência à Saúde Pública/Coletiva em pelo menos uma das bases consultadas.

Convém considerar, por outro lado, que existem periódicos científicos nacionais que abordam assuntos pertinentes à Saúde Pública, mas que não se reportam a essa temática nas bases de dados, pois têm como registro, para o item assunto/tema, a denominação da área específica. Um exemplo disso é a não-inclusão, aqui, de vários periódicos de enfermagem. Tal questão reflete, o que é também apontado por Coimbra Jr. ${ }^{2}$, a diversidade de disciplinas constitutivas da Saúde Pública Latino-americana, o que gera, igualmente, grande desafio a editores de revistas dessa área. Esse mesmo autor, como exemplo, menciona a ocorrência, não rara, num mesmo fascículo, de artigos em ciências sociais, epidemiologia, avaliação nutricional, microbiologia de alimentos, toxicologia, política de medicamentos e controle de vetores, entre outros. Tal fato foi também o determinante da necessidade de ampliar a presente pesquisa para outras bases além do Catálogo de Revistas de Saúde da BVS. Para isso, optou-se por estender a busca ao CCN e portal Latindex, em razão da verificação prévia dessas bases apresentarem maior número de títulos nacionais e os itens de informação requeridos para o presente trabalho.

A Lista de Periódicos Indexados na Base Lilacs e a Base de Dados de ISSN foram utilizadas como fontes complementares na recuperação de títulos ou outros dados. As listas de classificação Qualis-CAPES foram inicialmente utilizadas no intuito de recuperar o nível classifica- 
Revistas científicas nacionais referentes à temática Saúde Pública ou Saúde Coletiva, editadas entre 1998-2003, identificadas por consulta ao Catálogo de Revistas da BVS, e/ou ao CCN referente à área de Saúde Pública ou Saúde Coletiva e áreas afins, e/ou à base catálogo do Latindex, e/ou à lista de classificação de periódicos Qualis-CAPES, com respectivo número de registro ISSN, denominação da entidade responsável com sua localização regional.

\begin{tabular}{|c|c|c|c|c|c|c|c|c|}
\hline \multirow[b]{2}{*}{ № } & \multirow{2}{*}{$\begin{array}{l}\text { Revista } \\
\text { Título }\end{array}$} & \multirow[b]{2}{*}{ ISSN } & \multirow[b]{2}{*}{ BVS } & \multicolumn{2}{|c|}{ Fonte consultada } & \multirow[b]{2}{*}{ CAPES } & \multirow{2}{*}{$\begin{array}{l}\text { Responsabilidade } \\
\text { Entidade }\end{array}$} & \multirow[b]{2}{*}{ Local } \\
\hline & & & & $\mathrm{CCN}$ & Latindex & & & \\
\hline 1 & Ação Coletiva & $s / r$ & & $x$ & & & $\begin{array}{l}\text { Associação de Odontologia em Saúde } \\
\text { Coletiva (Brasília) }\end{array}$ & DF \\
\hline 2 & Alimentação e Nutrição & 0101-0103 & $x$ & $(X)$ & $(X)$ & & $\begin{array}{l}\text { Federação Brasileira das Associações } \\
\text { de Nutricionistas }\end{array}$ & SP \\
\hline 3 & Bio (Rio de Janeiro) & 0103-5134 & & $(X)$ & $x$ & & $\begin{array}{l}\text { Associação Brasileira de Engenharia } \\
\text { Sanitária e Ambiental }\end{array}$ & RJ \\
\hline 4 & Bioética & 0104-1401 & $x$ & $(X)$ & $(X)$ & $x$ & Conselho Federal de Medicina (Brasília) & DF \\
\hline 5 & Biosaúde & $1517-9664$ & & & $\mathrm{X}$ & & Universidade Estadual de Londrina & PR \\
\hline 6 & $\begin{array}{l}\text { BIS (Boletim do Instituto } \\
\text { de Saúde) }\end{array}$ & $1518-1812$ & & & $x$ & & Instituto de Saúde & SP \\
\hline 7 & $\begin{array}{l}\text { Boletim Bibliográfico/Serviço } \\
\text { de Biblioteca e Documentacão, } \\
\text { Faculdade de Saúde Pública, } \\
\text { Universidade de São Paulo }\end{array}$ & $0102-6305$ & & $x$ & & & Universidade de São Paulo & SP \\
\hline 8 & $\begin{array}{l}\text { Boletim do Centro de Pesquisa } \\
\text { e Processamento de Alimentos }\end{array}$ & $0102-0323$ & $x$ & $(X)$ & $(X)$ & $(X)$ & Universidade Federal do Paraná & PR \\
\hline 9 & Boletim Epidemiológico & 0374-6119 & $x$ & $(X)$ & $x$ & & Ministério da Saúde & RJ \\
\hline 10 & Cadernos de Saúde & $1415-6741$ & & & $x$ & $(X)$ & Faculdade de Leme, Universidade de Cuiabá & MT \\
\hline 11 & Cadernos de Saúde Pública & $0102-311 x$ & $x$ & $x$ & $x$ & $\mathrm{x}$ & $\begin{array}{l}\text { Escola Nacional de Saúde Pública, Fundação } \\
\text { Oswaldo Cruz, Ministério da Saúde }\end{array}$ & RJ \\
\hline 12 & $\begin{array}{l}\text { Cadernos do CCM (Centro } \\
\text { de Ciências Médicas) }\end{array}$ & $1413-5779$ & & & $x$ & & Universidade Federal Fluminense (Niterói) & RJ \\
\hline 13 & Cadernos Fundap & $0101-3211$ & $x$ & $(X)$ & $(X)$ & & Fundação do Desenvolvimento & SP \\
\hline & & & & & & & Administrativo & \\
\hline 14 & $\begin{array}{l}\text { Cadernos Saúde Coletiva } \\
\text { (Rio de Janeiro) }\end{array}$ & $1414-462 x$ & & $x$ & & $x$ & $\begin{array}{l}\text { Núcleo de Estudos em Saúde Coletiva, } \\
\text { Universidade Federal do Rio de Janeiro }\end{array}$ & RJ \\
\hline 15 & Ciência \& Saúde Coletiva & $1413-8123$ & $x$ & $(X)$ & $x$ & $x$ & $\begin{array}{l}\text { Associação Brasileira de Pós-graduação } \\
\text { em Saúde Coletiva }\end{array}$ & RJ \\
\hline 16 & Dados & $0011-5258$ & $x$ & $(X)$ & $(X)$ & $(X)$ & Instituto Universitário de Pesquisa & RJ \\
\hline 17 & $\begin{array}{l}\text { Divulgação (ou Divulgação } \\
\text { em Saúde para Debate) }\end{array}$ & $0103-4383$ & $x$ & $(X)$ & & $x$ & $\begin{array}{l}\text { Centro Brasileiro de Estudos de Saúde } \\
\text { (Londrina) }\end{array}$ & PR \\
\hline 18 & $\begin{array}{l}\text { DST - Jornal Brasileiro de } \\
\text { Doenças Sexualmente } \\
\text { Transmissíveis }\end{array}$ & 0103-4065 & & $(X)$ & $x$ & $x$ & $\begin{array}{l}\text { Sociedade Brasileira de Doenças } \\
\text { Sexualmente Transmissíveis }\end{array}$ & RJ \\
\hline 19 & Espaço para a Saúde (On-line) & $1517-7130$ & & & $x$ & $\mathrm{x}$ & Núcleo de Estudos em Saúde Coletiva & PR \\
\hline 20 & $\begin{array}{l}\text { Família, Saúde e } \\
\text { Desenvolvimento }\end{array}$ & $1517-6533$ & & & $x$ & $(X)$ & $\begin{array}{l}\text { Departamento de Enfermagem, } \\
\text { Universidade Federal do Paraná }\end{array}$ & PR \\
\hline 21 & $\begin{array}{l}\text { Higiene Alimentar (ou Revista } \\
\text { Higiene Alimentar) }\end{array}$ & 0101-9171 & $x$ & $(X)$ & $(X)$ & $(X)$ & D. P. : Impress & SP \\
\hline 22 & História, Ciências, Saúde & 0104-5970 & $x$ & $(X)$ & $(X)$ & $(X)$ & $\begin{array}{l}\text { Casa de Oswaldo Cruz, Fundação } \\
\text { Oswaldo Cruz }\end{array}$ & RJ \\
\hline 23 & $\begin{array}{l}\text { Informe Epidemiológico } \\
\text { do SUS }\end{array}$ & 0104-1673 & $x$ & $(X)$ & $(X)$ & $x$ & $\begin{array}{l}\text { Fundação Nacional de Saúde, Ministério } \\
\text { da Saúde }\end{array}$ & DF \\
\hline 24 & $\begin{array}{l}\text { Investigación en Sistemas } \\
\text { y Servicios de Salud } \\
\text { Cuadernos para Discusión }\end{array}$ & $1519-1990$ & & & $x$ & & $\begin{array}{l}\text { Rede de Investigação e Serviços de Saúde } \\
\text { no Cone Sul }\end{array}$ & RJ \\
\hline 25 & $\begin{array}{l}\text { Memórias do Instituto } \\
\text { Oswaldo Cruz }\end{array}$ & $0074-0276$ & $x$ & $(X)$ & $x$ & $x$ & $\begin{array}{l}\text { Fundação Oswaldo Cruz, Ministério } \\
\text { da Saúde }\end{array}$ & RJ \\
\hline 26 & $\begin{array}{l}\text { Momento \& Perspectiva } \\
\text { em Saúde }\end{array}$ & 0102-9398 & $x$ & $(X)$ & & $(X)$ & Grupo Hospitalar Conceição & RS \\
\hline 27 & Nutri Vitae & $1415-1081$ & & & $x$ & & Universidade de São Marcos & SP \\
\hline 28 & O Mundo da Saúde & 0104-7809 & $x$ & $(X)$ & & $x$ & Centro Universitário São Camilo & SP \\
\hline
\end{tabular}

(continua) 
Tabela 1 (continuação)

\begin{tabular}{|c|c|c|c|c|c|c|c|c|}
\hline \multirow[b]{2}{*}{ № } & \multirow{2}{*}{$\begin{array}{l}\text { Revista } \\
\text { Título }\end{array}$} & \multicolumn{5}{|c|}{ Fonte consultada } & \multirow{2}{*}{$\begin{array}{l}\text { Responsabilidade } \\
\text { Entidade }\end{array}$} & \multirow[b]{2}{*}{ Loca } \\
\hline & & ISSN & BVS & $\mathrm{CCN}$ & Latindex & CAPES & & \\
\hline 29 & Pesquisa em Saúde & 1518-7799 & & & $x$ & $(X)$ & Conhecimento \& Ciência Ltda. & PA \\
\hline 30 & Physis & 0103-7331 & $x$ & & $(X)$ & $x$ & $\begin{array}{l}\text { Instituto de Medicina Social, } \\
\text { Universidade do Estado do Rio de Janeiro }\end{array}$ & RJ \\
\hline 31 & $\begin{array}{l}\text { Planejamento e Políticas } \\
\text { Públicas }\end{array}$ & 0103-4138 & $x$ & $(X)$ & $(X)$ & $(X)$ & Instituto de Pesquisa Econômica Aplicada & DF \\
\hline 32 & Promoção da Saúde & 1517-1167 & & $x$ & & & $\begin{array}{l}\text { Secretaria de Políticas de Saúde, } \\
\text { Ministério da Saúde }\end{array}$ & DF \\
\hline 33 & $\begin{array}{l}\text { RAM (Revista da Administração } \\
\text { Municipal) }\end{array}$ & 0034-7604 & $x$ & $(X)$ & $(X)$ & $x$ & Universidade Federal de Pelotas & RS \\
\hline 34 & $\begin{array}{l}\text { RASPP (Revista da Associação } \\
\text { de Saúde Pública do Piauí) }\end{array}$ & $1415-5583$ & $x$ & & & & Associação de Saúde Pública do Piauí & $\mathrm{PI}$ \\
\hline 35 & $\begin{array}{l}\text { Revista Baiana de Saúde } \\
\text { Pública }\end{array}$ & 0100-0233 & $x$ & $(X)$ & $x$ & $(X)$ & Secretaria de Saúde do Estado da Bahia & BA \\
\hline 36 & $\begin{array}{l}\text { Revista Brasileira Ciências } \\
\text { da Saúde }\end{array}$ & $1415-2177$ & & $(X)$ & $x$ & $(X)$ & Universidade Federal da Paraíba & PB \\
\hline 37 & $\begin{array}{l}\text { Revista Brasileira de Educação } \\
\text { Médica }\end{array}$ & 0100-5502 & $x$ & $(X)$ & $(X)$ & $x$ & Associação Brasileira de Educação Médica & RJ \\
\hline 38 & $\begin{array}{l}\text { Revista Brasileira de } \\
\text { Epidemiologia }\end{array}$ & $1415-790 x$ & $x$ & $x$ & $(X)$ & $x$ & $\begin{array}{l}\text { Associação Brasileira de Pós-graduação } \\
\text { em Saúde Coletiva }\end{array}$ & RJ \\
\hline 39 & $\begin{array}{l}\text { Revista Brasileira de Nutrição } \\
\text { Clínica }\end{array}$ & 0103-7196 & $x$ & & $(X)$ & $x$ & $\begin{array}{l}\text { Sociedade Brasileira de Nutrição } \\
\text { Parenteral e Enteral (Porto Alegre) }\end{array}$ & RS \\
\hline 40 & $\begin{array}{l}\text { Revista Brasileira de } \\
\text { Odontologia em Saúde } \\
\text { Coletiva }\end{array}$ & $1518-0492$ & & $x$ & $(X)$ & $(X)$ & $\begin{array}{l}\text { Associação Brasileira de Saúde Bucal } \\
\text { Coletiva }\end{array}$ & DF \\
\hline 41 & $\begin{array}{l}\text { Revista Brasileira de Psiquiatria } \\
\text { (São Paulo) }\end{array}$ & $1516-4446$ & & $(X)$ & $x$ & $(X)$ & Associação Brasileira de Psiquiatria & SP \\
\hline 42 & $\begin{array}{l}\text { Revista Brasileira de Saúde } \\
\text { Materno Infantil }\end{array}$ & 1519-3829 & & $(X)$ & $x$ & $x$ & Instituto Materno Infantil de Pernambuco & PE \\
\hline 43 & $\begin{array}{l}\text { Revista Brasileira de Saúde } \\
\text { Ocupacional }\end{array}$ & 0303-7657 & $x$ & $(X)$ & $x$ & $x$ & $\begin{array}{l}\text { Fundação Centro Nacional de Segurança, } \\
\text { Higiene e Medicina do Trabalho }\end{array}$ & SP \\
\hline 44 & $\begin{array}{l}\text { Revista de Administração } \\
\text { Pública }\end{array}$ & 0034-7612 & $x$ & $(X)$ & $(X)$ & $x$ & $\begin{array}{l}\text { Escola Brasileira de Administração } \\
\text { Pública, Fundação Getúlio Vargas }\end{array}$ & RJ \\
\hline 45 & Revista de APS & $1516-7704$ & & & $x$ & $(X)$ & Universidade Federal de Juiz de Fora & MG \\
\hline 46 & Revista de Nutrição & $1415-5273$ & $x$ & $(X)$ & $(X)$ & $x$ & $\begin{array}{l}\text { Faculdade de Ciências Médicas, Pontifícia } \\
\text { Universidade Católica de Campinas }\end{array}$ & SP \\
\hline 47 & $\begin{array}{l}\text { Revista de Saúde do Distrito } \\
\text { Federal }\end{array}$ & $0103-4480$ & $x$ & $(X)$ & $x$ & $(X)$ & $\begin{array}{l}\text { Fundação do Ensino e Pesquisa em } \\
\text { Ciências da Saúde, Secretaria da Saúde } \\
\text { do Distrito Federal }\end{array}$ & DF \\
\hline 48 & Revista de Saúde Pública & 0034-8910 & $x$ & $(X)$ & $x$ & $x$ & $\begin{array}{l}\text { Faculdade de Saúde Pública, } \\
\text { Universidade de São Paulo }\end{array}$ & SP \\
\hline 49 & Revista do Instituto Adolfo Lutz & 0073-9855 & $x$ & $(X)$ & $(X)$ & $x$ & Sociedade de Estudos de Saúde & SP \\
\hline 50 & Revista Médica do lamspe & $0100-2872$ & & $(X)$ & $x$ & & $\begin{array}{l}\text { Hospital do Servidor Público Estadual } \\
\text { de São Paulo }\end{array}$ & SP \\
\hline 51 & $\begin{array}{l}\text { Revista Saúde e Ambiente } \\
\text { (Cuiabá) }\end{array}$ & $1516-5787$ & & & $x$ & $(X)$ & Universidade Federal do Mato Grosso & MT \\
\hline 52 & $\begin{array}{l}\text { Revista Saúde e Ambiente } \\
\text { (Joinville) }\end{array}$ & $1518-756 x$ & & $(X)$ & $x$ & $(X)$ & Universidade da Região de Joinville & SC \\
\hline 53 & Salusvita (ou Revista Salusvita) & 0101-9910 & & $(X)$ & $x$ & $(X)$ & Universidade do Sagrado Coração & SP \\
\hline 54 & São Paulo Medical Journal & 0035-0362 & & $(X)$ & $x$ & $x$ & Associação Paulista de Medicina & SP \\
\hline 55 & Saúde (Porto Alegre) & $1413-8816$ & & $x$ & & & Universidade Federal do Rio Grande do Sul & RS \\
\hline 56 & Saúde (Santa Maria) & 0103-4952 & $x$ & $(X)$ & & & $\begin{array}{l}\text { Centro de Ciências da Saúde, Universidade } \\
\text { Federal de Santa Maria }\end{array}$ & RS \\
\hline 57 & Saúde e Cidade em Debate & $1516-7267$ & & & $x$ & & $\begin{array}{l}\text { Conselho Nacional das Secretarias } \\
\text { Municipais de Saúde }\end{array}$ & RJ \\
\hline 58 & Saúde e Sociedade & 0104-1290 & $x$ & $(X)$ & & $x$ & Universidade de São Paulo & SP \\
\hline 59 & Saúde em Debate & 0103-1104 & $x$ & $(X)$ & $x$ & $x$ & Centro Brasileiro de Estudos em Saúde & RJ \\
\hline
\end{tabular}

(continua) 
Tabela 1 (continuação)

\begin{tabular}{|c|c|c|c|c|c|c|c|c|}
\hline \multirow[b]{2}{*}{ № } & \multirow{2}{*}{$\begin{array}{l}\text { Revista } \\
\text { Título }\end{array}$} & \multicolumn{5}{|c|}{ Fonte consultada } & \multirow{2}{*}{$\begin{array}{l}\text { Responsabilidade } \\
\text { Entidade }\end{array}$} & \multirow[b]{2}{*}{ Local } \\
\hline & & ISSN & BVS & $\mathrm{CCN}$ & Latindex & CAPES & & \\
\hline 60 & Saúde em Foco & $1519-5600$ & & & $x$ & $x$ & $\begin{array}{l}\text { Secretaria Municipal de Saúde } \\
\text { do Rio de Janeiro }\end{array}$ & RJ \\
\hline 61 & Saúde on line & $1415-6474$ & & & $x$ & & $\begin{array}{l}\text { Hospital do Andaraí, Instituto Nacional } \\
\text { de Previdência Social }\end{array}$ & RJ \\
\hline 62 & $\begin{array}{l}\text { Saúde, Sexo e Educação (ou } \\
\text { Revista Saúde, Sexo e Educação) }\end{array}$ & $1415-6547$ & & $(X)$ & $x$ & $x$ & $\begin{array}{l}\text { Instituto Brasileiro de Medicina } \\
\text { de Reabilitação }\end{array}$ & RJ \\
\hline 63 & Semina & $0101-3742$ & & $(X)$ & $x$ & $(X)$ & Universidade Estadual de Londrina & PR \\
\hline 64 & $\begin{array}{l}\text { SOS. Saúde Ocupacional } \\
\text { e Segurança }\end{array}$ & $0100-8684$ & & $(X)$ & $x$ & & $\begin{array}{l}\text { Associação Brasileira para Prevenção } \\
\text { de Acidentes }\end{array}$ & $\mathrm{SP}$ \\
\hline 65 & Temas em Saúde & $1519-0870$ & & & $x$ & $(X)$ & Carlos Bezerra de Lima (João Pessoa) & PB \\
\hline 66 & $\begin{array}{l}\text { The Brazilian Journal of } \\
\text { Infectious Diseases }\end{array}$ & $1413-8670$ & $x$ & $(X)$ & $(X)$ & $x$ & $\begin{array}{l}\text { Brazilian Society of Infectious Diseases } \\
\text { (Salvador) }\end{array}$ & BA \\
\hline
\end{tabular}

Legenda: $\mathrm{s} / \mathrm{r}=$ sem registro; $\mathrm{X}=$ referida à especialidade/assunto/tema: Saúde Pública ou Saúde Coletiva;

$(X)=$ referida à especialidade/assunto/tema de denominação diversa de Saúde Pública ou Saúde Coletiva.

tório dos periódicos, dada a repercussão dessa classificação nas avaliações de produtividade científica do meio acadêmico. Diante de divergências encontradas entre essas listas e o recebimento da informação de estar ocorrendo reexame da classificação dos periódicos nas instâncias responsáveis da CAPES, foram utilizadas para o presente estudo apenas indicações, dessa fonte, para verificar a existência dos títulos, respectivo ISSN e sua referência, ou não, à Saúde Coletiva, na lista geral Qualis-2001 da CAPES, que abrange 18.360 títulos (alguns repetidos) entre nacionais e estrangeiros. Postergou-se assim a pretendida análise quanto à classificação desses periódicos para um trabalho futuro.

Sobre o tema da situação de vigência do periódico, foi estabelecido o período de 1998 a 2003 para evitar exclusão de títulos por eventuais falhas quanto à sua circulação, problema que afeta parte do meio editorial brasileiro, mesmo quando situado em grandes centros. Para isso considerou-se também o fato, conforme mencionado por Targino \& Garcia 3 , de que periódicos novos levam em média até cinco anos para se firmarem em suas especialidades. Esses autores analisam ainda que a própria situação, periodicidade e regularidade interferem no processo de editoração, distribuição e circulação da revista. Parece pertinente acrescentar o comentário de que esses aspectos, entre outros, conforme apontados por Krzyzanowski 4 , fazem das revistas científicas brasileiras alvo de críticas, em âmbito internacional, e conseqüente ausência de indexação nos índices e bibliografias.

Por outro lado, diante de lamentáveis lacunas ou discrepâncias de registro que ocorrem por vezes nas fontes consultadas, ora referentes a um dos itens pesquisados, ora a outro, foram utilizados meios de busca via Google, ou mesmo acesso físico às revistas como alternativa para completar ou confrontar dados.

De acordo com as quantificações resumidas na Figura 1, 56,1\% dos periódicos identificados referem-se à Saúde Pública/Coletiva em apenas uma das cinco fontes; $34,8 \%$ têm indicação para essa temática em duas das bases pesquisadas; e 7,6\% têm essa referência em três das fontes. Apenas uma das revistas $(1,5 \%)$ se refere à Saúde Pública em todas as bases consultadas.

De acordo com a Tabela 1, dos 66 títulos identificados, 55 têm indicadores bibliográficos na base Latindex, dos quais 36 se referem à Saúde Pública/Coletiva. Assim, essa é a base com maior número de inclusões de títulos pertinentes a este estudo. No CCN, foram localizados registros para 48 títulos, dos quais oito referentes à Saúde Pública. Pela BVS foram recuperados 33 títulos. Na Lista de Classificação da CAPES, 47 periódicos, dos quais 27 relativos à Saúde Coletiva. Por esses dados, verifica-se que a maioria das 66 revistas identificadas detém registros em mais de uma das fontes pesquisadas, não estando necessariamente remetidas para a mesma área/especialidade/assunto nas diferentes fontes consultadas.

Entretanto, 13 revistas possuem registros em apenas uma das fontes consultadas. Essas e outras três revistas se remetem apenas à Saúde Pública/Coletiva. Portanto, cinqüenta revistas se referem também a outras áreas.

Quanto ao levantamento para identificar o vínculo institucional da responsabilidade editorial das revistas e correspondente localização regional, foi verificado um perfil de diferenças 
Quantidade de periódicos nacionais referentes à Saúde Pública ou Saúde Coletiva, editados entre 1998 e 2003,

e sua distribuição pelo número de registros por fonte consultada.

Quantidade de periódicos referentes à Saúde Pública/

Coletiva em cada fonte consultada

Número de periódicos

\begin{tabular}{|c|}
\hline 36 \\
8 \\
33 \\
27 \\
\hline
\end{tabular}

Quantidade de periódicos referentes à Saúde Pública/ Coletiva que tem essa indicação em uma ou mais das fontes consultadas

Número de periódicos

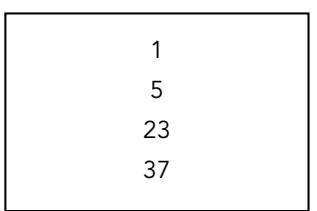

Número de fontes

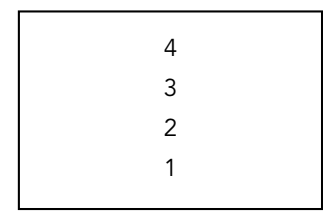

no que diz respeito à natureza jurídica dessas entidades. Quanto à sua localização geográfica, compreende 13 Unidades da Federação. São elas, conforme apontadas na Tabela 1: Rio de Janeiro, São Paulo, Distrito Federal, Rio Grande do Sul, Paraná, Paraíba, Bahia, Mato Grosso, Santa Catarina, Minas Gerais, Piauí, Pernambuco e Pará. O número de títulos de revistas e as correspondentes localizações das entidades responsáveis estão representados na Figura 2.

A Figura 2 mostra uma distribuição comparativa em que Rio de Janeiro (com 20 revistas) e São Paulo (com 17) contribuem com mais da metade dos títulos editados. Seguem-se o Distrito Federal (com 7), Rio Grande do Sul e Paraná (cada um com 5), Paraíba (com 3), Bahia e Mato Grosso (cada um com 2), Santa Catarina, Minas Gerais, Piauí, Pernambuco e Pará (cada um com 1).

Para uma configuração gráfica da distribuição dos locais de sede das entidades responsáveis, considerando-se as regiões geopolíticas, tem-se representada, na Figura 3, a correspondente distribuição proporcional do número de títulos.

A Figura 3 ilustra, por ordem decrescente do porcentual de títulos, a Região Sudeste, que concentra $57,6 \%$ das entidades responsáveis; a Região Sul, com 16,7\% das revistas; a CentroOeste, com 13,6\%; a Nordeste, com $10,6 \%$ e a Região Norte, com 1,5\% dos títulos editados. Em trabalhos de avaliação relativos a periódicos nacionais, como também com referência à presença destes em bases internacionais, verificam-se discussões sobre temas relacionados à natureza jurídica da responsabilidade editorial das revistas.
Targino \& Garcia 3, analisando periódicos brasileiros constantes da base de dados do Institute for Scientific Information (ISI), apontam a participação majoritária de sociedades científicas e associações profissionais em contraposição com o aporte de instituições universitárias. Apontam também relações dessas presenças com subsídios que as instituições recebem para fins de editoração de suas revistas, assim como com a origem regional desses periódicos, a qual é similar ao quadro aqui levantado. Isto é, concentração na Região Sudeste, seguida da Região Sul, e as instituições de ensino superior representando um terço das entidades responsáveis.

Um estudo da Fundação de Amparo à Pesquisa do Estado de São Paulo - FAPESP - (http: //www2.fapesp.br/indct/cap06/cap06.htm, acessado em 25/Abr/2003), também sobre publicações brasileiras indexadas na base ISI, aponta que a maioria desses trabalhos originase de apenas dez campus universitários. Portanto, tem-se natureza jurídica relativa à origem da pesquisa diversa da natureza jurídica da entidade de origem do periódico em que é divulgada.

Por outro lado, considere-se que, conforme menciona Miranda 5 (p. 1), dentre as atividades interferentes na produção científica, pode-se entender também "as que contribuem para viabilizar um produto enquanto veículo (suporte físico) de comunicação do conhecimento". Assim, considerando a inter-relação da produção do conhecimento e o periódico que a veicula, seria de interesse que questões como as acima apresentadas viessem a ser estudadas em con- 
Figura 2

Distribuição do número de revistas científicas nacionais, com circulação entre 1998 e 2003, referentes à temática Saúde Pública ou Saúde Coletiva, em relação a localização (Unidade da Federação) da entidade responsável.

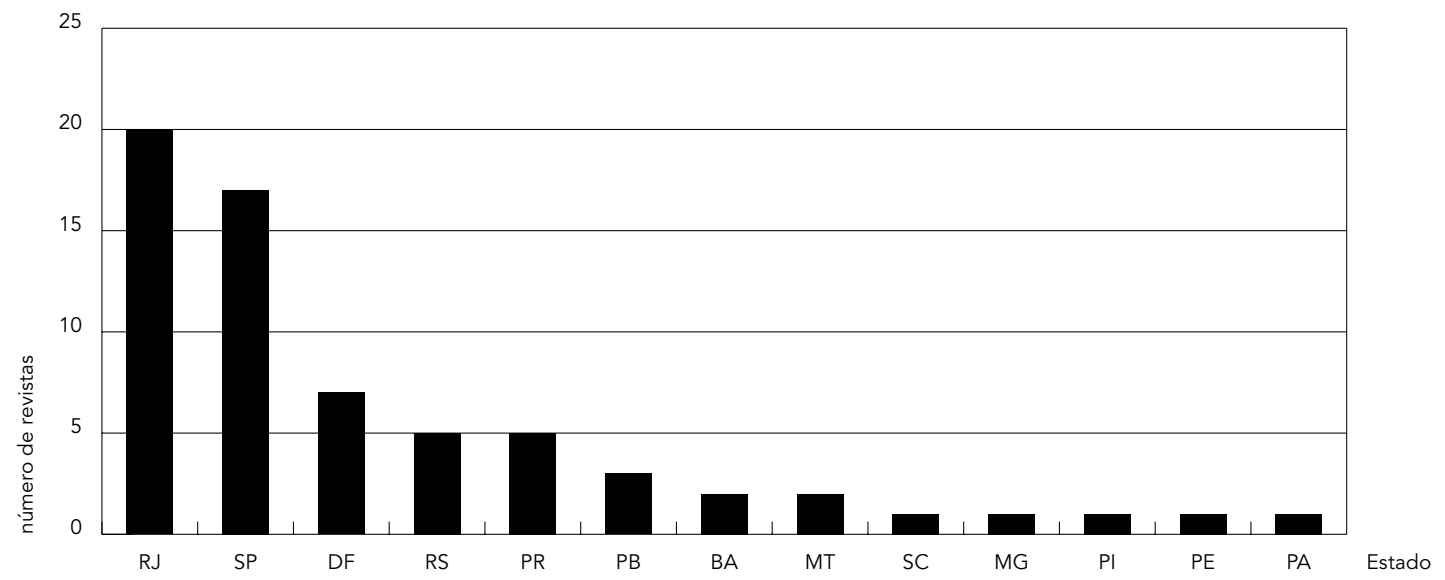

Figura 3

Distribuição percentual das revistas científicas nacionais com circulação entre 1998 e 2003, referentes à temática de Saúde Pública ou Saúde Coletiva, de acordo com a região de localização da entidade responsável.

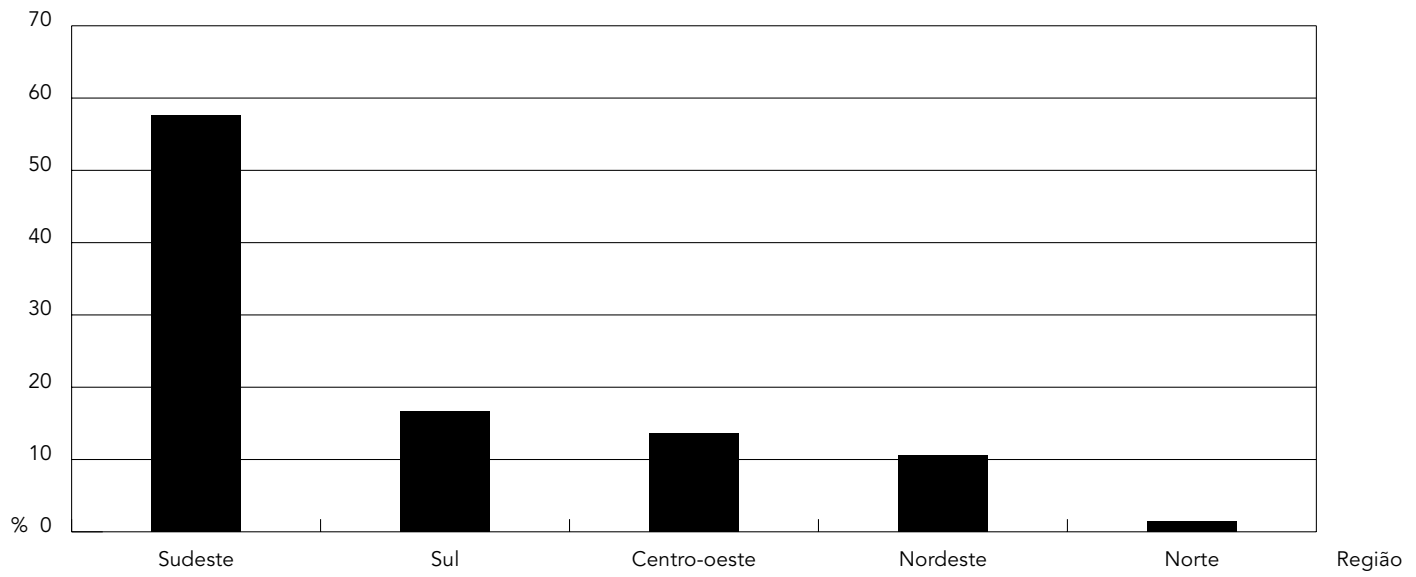

formidade com o elenco de revistas referentes à Saúde Pública/Coletiva, bem como fosse feito um levantamento de dados adicionais que permitisse uma análise quanto à situação das mesmas frente aos parâmetros mencionados. Tal extensão de estudo poderia subsidiar sugestões de política e fomento relacionadas à problemática da divulgação científica na área da Saúde Pública, com vistas a uma crescente visibilidade, qualidade e conseqüente posicionamento da produção científica nacional.

\section{Conclusões}

Foram identificados 66 títulos de periódicos científicos nacionais para os quais há indicação de editoração dentro do período 1998-2003, 
e que se referem à Saúde Pública e/ou Saúde Coletiva em pelo menos uma das fontes pesquisadas (BVS, CCN, Latindex e CAPES).

A maioria desses títulos é remetida, em uma ou mais das bases consultadas, a áreas da Saúde de denominação diferente.

As instituições de vínculo da responsabilidade editorial desse elenco de revistas corresponde a entidades de diversas naturezas jurídicas e de 13 Unidades da Federação, com concentração na Região Sudeste, em que Rio de Janeiro e São Paulo são os estados de maior participação.

\section{Colaboradores}

G. Rummler redigiu o artigo com supervisão de A. W. P. Spínola.

\section{Agradecimentos}

A Ângela Maria Belloni Cuenca, Maria do Carmo Avamilano Alvarez e Maria Lúcia Evangelista de Faria Ferraz pela receptividade e ajuda inicial na utilização dos meios de acesso a fontes de consulta da Biblioteca da Faculdade de Saúde Pública da Universidade de São Paulo; a Suely Maffia e Cristine Boianovsky, do Centro Brasileiro do ISSN, pela disponibilização de arquivos de registro do ISSN; a Edilma Barros Macedo e Lúcia Alves Ferreira e Silva, da Coordenação e Aperfeiçoamento de Pessoal do Ensino Superior, pela disponibilização de listas de periódicos Qualis-CAPES, utilizadas aqui como apoio informativo; a Marcos Amorim, da BIREME, pelo fornecimento de endereços de editoras de algumas revistas pesquisadas neste trabalho; a Viviane Rummler da Silva, pela paciente contribuição na lida de meios de informática e digitações, assim como, à Profa. Egle Emídia Guerzoni Piva e Liane Inês Constantino Brandimarti, pela revisão gramatical do texto. Apoio financeiro: convênio Fundação de Amparo à Pesquisa da Bahia/UEFS no $249 / 2002$.

\section{Resumo}

A diversidade de conteúdos em periódicos científicos nacionais referentes à Saúde Coletiva ou à Saúde Pública reflete o aspecto peculiar da abrangência desse campo e das interfaces com diversas outras áreas. Foi elaborada uma pesquisa bibliométrica em cinco bases de dados, compreendendo catálogos e portais de revistas, com o objetivo de identificar os periódicos relativos a essa temática e editados dentro do período 19982003, além de estabelecer sua origem quanto à localização geográfica da entidade responsável. Por essa pesquisa foram listados, inicialmente, 118 títulos diferentes. Por confrontação e conjugação de indicadores dessas bases, a listagem ficou reduzida para 83 periódicos, sendo 66 com vigência dentro dos últimos cinco anos. Dessa listagem, 57,6\% têm a sua entidade responsável localizada na Região Sudeste; 16,7\%, na Região Sul; 13,6\%, na Centro-Oeste; 10,6\%, na Região Nordeste e 1,5\%, na Região Norte. Integram ainda os resultados deste estudo a apresentação, em Tabela, das revistas pelo correspondente título, ISSN, denominação da instituição responsável e respectiva localização regional.

Saúde Pública; Índice de Periódicos; Publicações Periódicas; Bibliometria

\section{Referências}

1. Lopes IL. Estratégia de busca na recuperação da informação: revisão da literatura. Ciência da Informação 2002; 31:60-71.

2. Coimbra Jr. CEA. Produção científica em Saúde Pública e as bases bibliográficas internacionais. Cad Saúde Pública 1999; 15:883-8.

3. Targino MG, Garcia JCR. Ciência brasileira na base de dados do Institute for Scientific Information (ISI). Ciência da Informação 2000; 29:103-17.

4. Krzyzanowski RF, Ferreira MCG. Avaliação de periódicos científicos e técnicos brasileiros. Ciência da Informação 1998; 27:165-75.

5. Miranda DB, Pereira MNF. O periódico científico como veículo de comunicação: uma revisão de literatura. Ciência da Informação 1996; 25:1-11.

Recebido em 17/Nov/2003

Versão final reapresentada em 26/Fev/2004

Aprovado em 05/Abr/2004 